\title{
To Compare The Effect Of Core Stability Exercises And Muscle Energy Techniques On Low Back Pain Patients.
}

\author{
Shivalika*, Apoorv Narain**, Jagmohan Singh***, Sabyasachi Bhowmik**** \\ ${ }^{* M P T} 2^{\text {nd }}$ Year Student, **Associate Professor, ${ }^{* * * P r o f e s s o r ~ a n d ~ P r i n c i p a l, ~ G i a n ~ S a g a r ~ C o l l e g e ~ o f ~}$ \\ Physiotherapy, ****Associate Professor (orthopaedics deptt.), Gian Sagar Medical College and Hospital, \\ Rajpura, Distt. Patiala, Punjab (INDIA).
}

\begin{abstract}
Low back pain is considered one of the commonest condition in the western and industrialized countries. It is estimated that up to 50\% of adults experience low back pain during their life span. People of all age group can be effected by this menace irrespective to their gender and quality of life. It has become one of the leading causes for the visit to physician thus also puts a heavy burden on the currency of the country. Physiotherapy is the most widely used form of treatment adopted for gaining relief from low back pain. The exercises include stretching, strengthening, range of motion exercises, McKenzie therapy and core stability exercises other techniques like muscle energy technique etc. It has been concluded in various studies core stability exercises and muscle energy technique are beneficial in low back pain patients but comparison of their effect needs to be established to provide early and better relief from the disability. Therefore objective of the study was to compare the effect of core stability exercises and muscle energy techniques on low back pain patients. 60 subjects aged $18-45$ years with low back pain were made part of the study based on inclusion and exclusion criteria and were then divided into three groups named A, B and C. Group A received core stability exercise and conventional physiotheraphy and group B received muscle energy techniques and conventional physiotherapy. The exercise program was given on alternate days with a total of 24 sessions and progression of the activity was made within the tolerance of the patient. Pre and post treatment readings were taken of pain, ROM and quality of life scale. Results were analyzed using paired, unpaired t-test and ANOVA. Results showed that there is significant effect on pain, ROM and quality of life scale in the three groups but group A was clinically more significant than the other groups. The study concluded that patients with low back pain are benefitted more by core stability exercises. So, core stability exercises should be practiced more.
\end{abstract}

Keywords: Low Back Pain, Core Stabilization Exercises, Muscle Energy Technique.

\section{Introduction}

Pain in the lower back is a common concern, affecting up to $90 \%$ of population at some point in their lifetime, up to $50 \%$ have more than one episode (William and Shiel, 2012). It has been found that annual expenditure on the low back pain range from $\$ 30-70$ billion (Driscoll, 2011). It is determined that the risk of back pain is twice as high once a history of the condition has been established (Hestbaek 2003). Population is facing number of obstacles in their daily life. Pain and muscle weakness are the most common obstacles in carrying out activities of daily living.

The main causative factor that can cause back pain is poor posture while sitting, standing and lifting heavy weights. Other factors that can cause low back pain include spinal disorders and systemic diseases. (Cox and Trierk 1987).

Physiotherapy is the most widely used form of treatment adopted for gaining relief from low back pain. It is used in both modes, as single line of treatment including exercises or in a form of combination with electrotherapy modalities like short wave diathermy, interferential therapy etc. The exercises include stretching, strengthening, range of motion exercises, McKenzie therapy and core stability exercises (Kumar, 2011). In order to improve low back pain there needs to be enough strength in abdominal and trunk muscles and the pelvic floor therefore strengthening exercises play an important role (Ferreira et al, 2006). Core stability exercises have become one of the fitness trend broadly used exercises for low back pain. Benefits of core stabilization have been rooted, from improving athletic performance and preventing injuries, to alleviating low back pain (Hodges, 1996). Lack of sufficient coordination in core musculature can lead to decreased efficiency of movement and compensatory patterns, causing strain and overuse injuries. There is ample evidence that individuals with low back pain and sacroiliac pain lack proper recruitment of core muscles and exhibit core weakness. There is also evidence of increased fatigue, decreased cross section, and fatty infiltration of paraspinal muscles in patients with chronic low back pain. Patients with back pain also seem to over-activate superficial global muscles whereas control and activation of the deep spinal muscles is impaired. Thus core stability exercises have strong theoretical basis for prevention of different musculoskeletal conditions and the treatment of spinal disorders (McGill, 2001). 
There are different techniques used to cure low back pain. Muscle energy technique was developed by Fred Mitchell, Sr. It involves the use of isotonic concentric or eccentric, contractions or a series of rhythmically pulsating contractions and isometric variations (Ruddy 1961 and Schmitt, 1999). It is useful in increasing extensibility and range of motion of various joints and provides good results in the patients with back pain (Hamilton, 2003). These techniques are used as the treatment of weakened muscles, restricted joints, using muscle effort to mobilize joints and tissues and also used as an evaluation procedure to identify and range of motion restriction (Tonelli, 2006).

It has been concluded in various studies that both core stability exercises and muscle energy technique are beneficial in low back pain patients. Comparison of their effect needs to be established to provide early and better relief from the disability.

\section{Need of the study}

Low back pain is the common disability for people and hinders their functional ability. Core stability exercise and muscle energy technique showed marked improvement. There is a need to compare both the treatment regime in order to provide better results in less time.

\section{Aim of the Study}

To compare the effect of core stability exercises and muscle energy techniques in low back pain patients.

\section{Objective of the study}

To evaluate the effect of core stability exercises on low back pain, range of motion and quality of life.

To evaluate the effect of muscle energy techniques on low back pain, range of motion and quality of life.

\section{Hypothesis}

\section{Null hypothesis}

There will be no significant difference in the effect of core stabilization exercises and muscle energy technique in patients with low back pain

\section{Alternate hypothesis}

There will be significant difference in the effect of core stabilization exercises and muscle energy techniques in patients with low back pain.

\section{Review of literature}

Muthukrishnan, Shenoy and Sandhu. 2010 did study to examine the differential effect of core stability exercise training and conventional physiotherapy regime on altered postural control parameters in patients with chronic low back pain (CLBP). As heterogeneity in CLBP population moderates the effect of intervention on outcomes, in this study, interventions approaches were used based on sub-groups of CLBP. On the basis of the study they concluded that core stability exercise group provide better result.

Noelle, Terry and Hertel. 2003 did a study that Muscle energy technique (MET) is a form of manual therapy frequently used to correct lumbopelvic pain (LPP), here in the patient voluntarily contracts specific muscles against the resistance of the clinician. Studies on MET regarding magnitude and duration of effectiveness are limited. This study was a randomized controlled trial in which 20 subjects with self-reported LPP were randomized into two groups (MET or control) after magnitude of pain was determined. MET of the hamstrings and iliopsoas consisted of four 5 -second hold/relax periods, while the control group received a no treatment. Tests for current and worst pain, and pain with provocation were administered at baseline, immediately following intervention and 24 hours after intervention.

Study Design: Experimental study design comparative in nature

Research setting: Out patient department of Physiotherapy, Gian Sagar Medical College and Hospital, Ram Nagar, Rajpura, Distt. Patiala.

Study duration: 6 months

Population Sample: 60 patients

Sampling technique: Random Sampling Technique

Inclusion criteria ( Osterbauer and DeVita, 1991): Age: 18-45 years. both males and females, patients with mechanical back pain, Patients with no medication related to back pain Back strain or sprain.

Exclusion criteria: History of any neurological disorders, subjects who are not cooperative to procedure, patients with cardiovascular disease, participating in another research study involving back, patients with diabetics, patients with ankylosing spondylitis, other significant spinal pathology, patients with neoplasms, patients with diseases of the viscera.

\section{Procedure}

60 subjects were made part of the study based on the inclusion and exclusion criteria. After taking consent, the subject will be randomly divided into three groups named A, B and C 20 in each group. Group A received 24 
sessions of core stabilization exercises 8 sessions per phase 3 times per week on alternate days along with conventional physiotherapy. Exercise program was done in 3 phases (McGill, 2001). Phase 1 included abdominal bracing, single knee to chest and bridging exercises. Phase 2 included abdominal strengthening, basic stabilization and wall squats with swiss ball. Phase 3 included lumbar extensor strengthening, lumbar extension on ball and corner stretch. Group B received 24 sessions of muscle energy technique 3 times per week alternate day along with conventional physiotherapy. It included isotonic contraction using post isometric relaxation and isotonic contraction using reciprocal inhibition. Group $\mathrm{C}$ was control group it received only conventional physiotherapy that is hot packs, stretching and strengthening of back (Kumar, 2011).

Dependent Variables : Visual analogue scale(VAS), Quality of life scale (Bruckhard and Anderson, 2003) and Goniometry

Independent Variable: Core Stabilization Exercises and Muscle Energy Technique

\section{Data analysis}

Results were analysed by paired, unpaired t- test and ANOVA. Paired t- test was applied between pre and post values of VAS, QOL and lumbar ROM of group A. Paired t- test was applied between pre and post values VAS, QOL and lumbar ROM of group B. Paired t- test was applied between pre and post values VAS, QOL and lumbar ROM of group C. Unpaired t- test was applied to the difference of pre and post values of group A with difference of pre and post values of group B. Unpaired t- test was applied to the difference of pre and post values of group A with difference of pre and post values of group C. Unpaired t- test was applied to the difference of pre and post values of group B with difference of pre and post values of group C. ANOVA was applied to the differences of post values of the three groups.

Table 1.1

Mean and standard deviation of pre-treatment, post-treatment and their difference of VAS score of the groups.

\begin{tabular}{|c|c|c|}
\hline GROUPS & PRE & POST \\
& & Mean \\
& Mean & \pm \\
& SD & SD \\
\hline & $7.3 \pm 0.801$ & $2.7 \pm 0.66$ \\
A & & \\
\hline B & $7.6 \pm 0.753$ & $4.65 \pm 0.74$ \\
\hline C & $7.4 \pm 0.882$ & $6.15 \pm 1.089$ \\
\hline
\end{tabular}

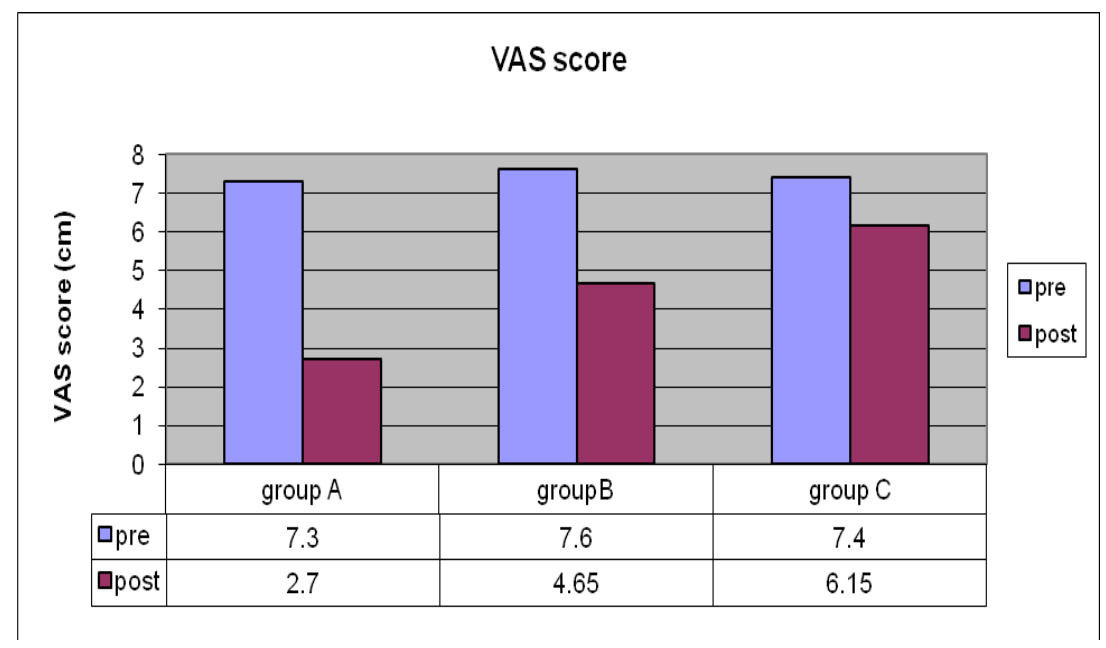

Graph 1.1

Graph represent mean of VAS at Pre, Post interval for the subjects of Group A, Group B and Group 
Table 1.2

Mean, SD and SE of QOL at Pre, Post and Mean diff. (Pre-Post) interval for the subjects of Group A, Group B and Group C

\begin{tabular}{|c|c|c|}
\hline & PRE & POST \\
\cline { 2 - 3 } & Mean & Mean \\
& \pm & \pm \\
GROUPS & SD & SD \\
\hline A & $6 \pm 0.858395$ & $1.3 \pm 0.470162$ \\
\hline B & $6.35 \pm 0.74516$ & $1.9 \pm 0.788069$ \\
\hline C & $6.25 \pm 0.71635$ & $2 \pm 0.725476$ \\
\hline
\end{tabular}

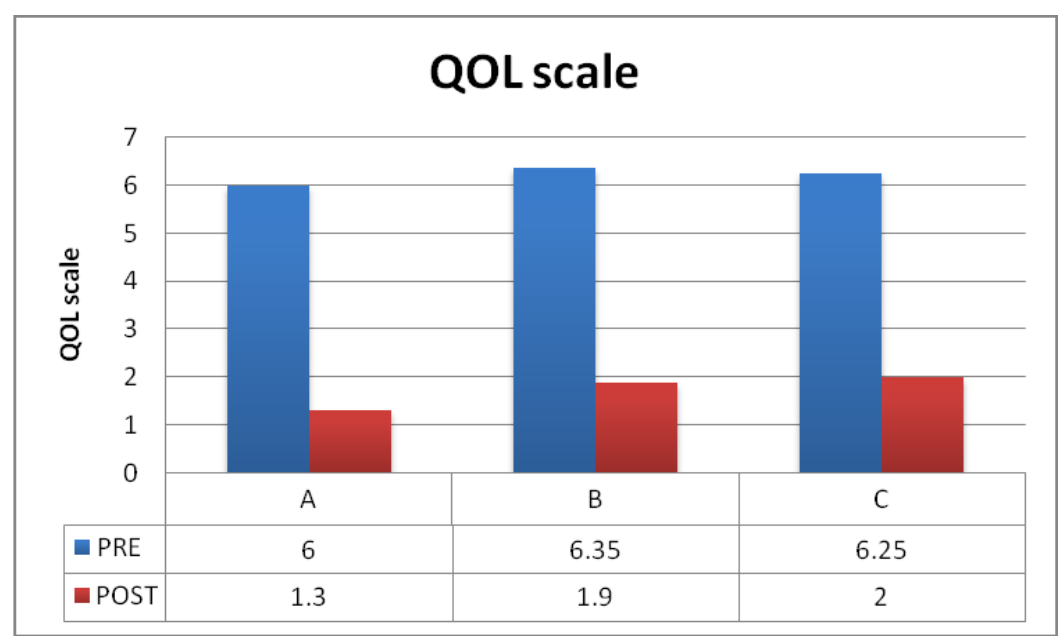

Graph 1.2

Graph represent mean of QOL at Pre, Post interval for the subjects of Group A, Group B and Group C

Table 1.3

Mean, SD and SE of ROM ( lumbar flexion) at Pre, Post and Mean diff. (Pre-Post) interval for the subjects of Group A, Group B and Group C

\begin{tabular}{|l|l|l|}
\hline \multirow{2}{*}{ GROUPS } & PRE & POST \\
\cline { 2 - 3 } & Mean & $\begin{array}{l}\text { Mean } \\
\pm\end{array}$ \\
& SD & SD \\
\hline A & $40.65 \pm 4.738$ & $54.2 \pm 3.427$ \\
\hline B & $40.45 \pm 6.395$ & $49 \pm 4.834$ \\
\hline C & $39.9 \pm 6.617$ & $47.75 \pm 5.665$ \\
\hline
\end{tabular}

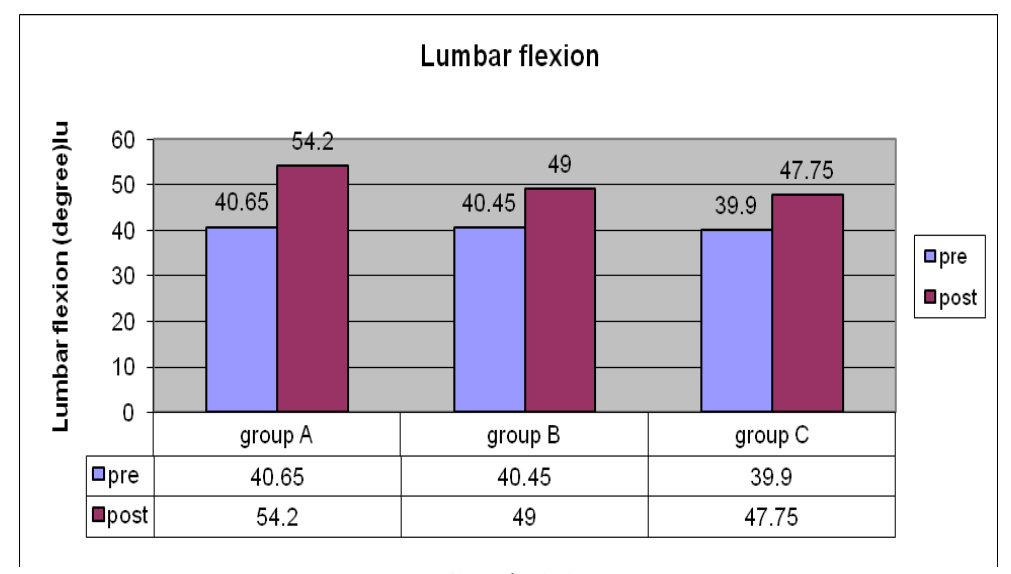

Graph 1.3

Graph represent Mean of lumbar flexion at Pre, Post interval for the subjects of Group A, Group B and Group C 
Table 1.4

Mean, SD and SE of ROM (lumbar extension) at pre, post and mean diff. (Pre-Post) interval for the subjects of Group A, Group B and Group C

\begin{tabular}{|l|l|l|}
\hline \multirow{2}{*}{ GROUPS } & PRE & POST \\
\cline { 2 - 3 } & $\begin{array}{l}\text { Mean } \\
\pm\end{array}$ & $\begin{array}{l}\text { Mean } \\
\pm\end{array}$ \\
SD & SD \\
\hline A & $13.4 \pm 3.5$ & $22.6 \pm 2.326$ \\
\hline B & $12.75 \pm 3.385$ & $19.15 \pm 3.166$ \\
\hline C & $14.1 \pm 4.115$ & $18.3 \pm 3.294$ \\
\hline
\end{tabular}

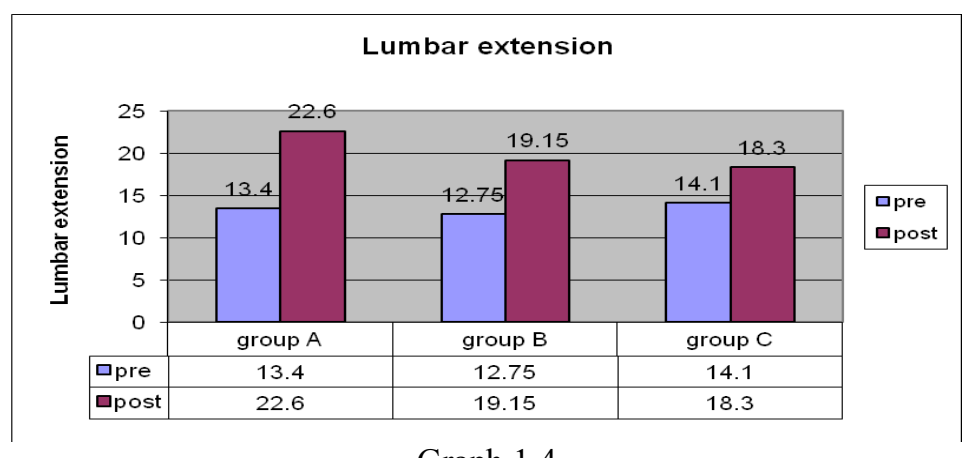

Graph 1.4

Graph represent Mean of lumbar extension at Pre, Post interval for the subjects of Group A, Group B and Group $\mathrm{C}$

Table 1.5

Mean, SD and SE of ROM (lumbar side flexion on right side) at Pre, Post and Mean diff. (Pre-Post) interval for the subjects of Group A, Group B and Group C

\begin{tabular}{|l|l|l|}
\hline \multirow{4}{*}{ GROUPS } & PRE & POST \\
\cline { 2 - 3 } & $\begin{array}{l}\text { Mean } \\
\pm\end{array}$ & $\begin{array}{l}\text { Mean } \\
\pm \\
\text { SD }\end{array}$ \\
\hline A & & \\
& $10.95 \pm 2.723$ & $21.1 \pm 2.174$ \\
\hline B & & \\
& $11.05 \pm 2.543$ & $17.65 \pm 2.455$ \\
\hline C & $11.35 \pm 2.978$ & $16.4 \pm 3.033$ \\
\hline
\end{tabular}

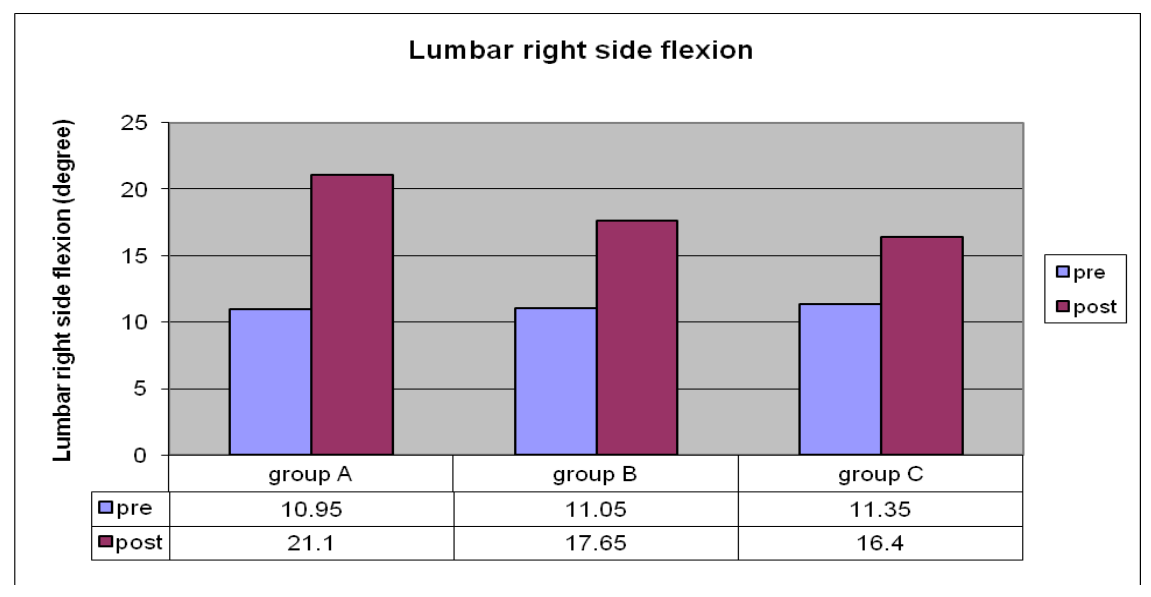

Graph 1.5

Graph represent Mean of lumbar flexion on right side at Pre, Post interval for the subjects of Group A, Group B and Group C 
Table 1.6

Mean, SD and SE of ROM (lumbar side flexion on left side) at Pre, Post and Mean diff. (Pre-Post) interval for the subjects of Group A, Group B and Group C

\begin{tabular}{|l|l|l|}
\hline \multirow{2}{*}{ GROUPS } & PRE & POST \\
\cline { 2 - 3 } & $\begin{array}{l}\text { Mean } \\
\pm\end{array}$ & $\begin{array}{l}\text { Mean } \\
\pm\end{array}$ \\
& SD & SD \\
\hline $\mathrm{A}$ & $11.5 \pm 2.564$ & $20.9 \pm 2.673$ \\
\hline $\mathrm{B}$ & $11.6 \pm 2.458$ & $18.25 \pm 2.221$ \\
\hline $\mathrm{C}$ & $11.1 \pm 2.978$ & $17.1 \pm 3.416$ \\
\hline
\end{tabular}

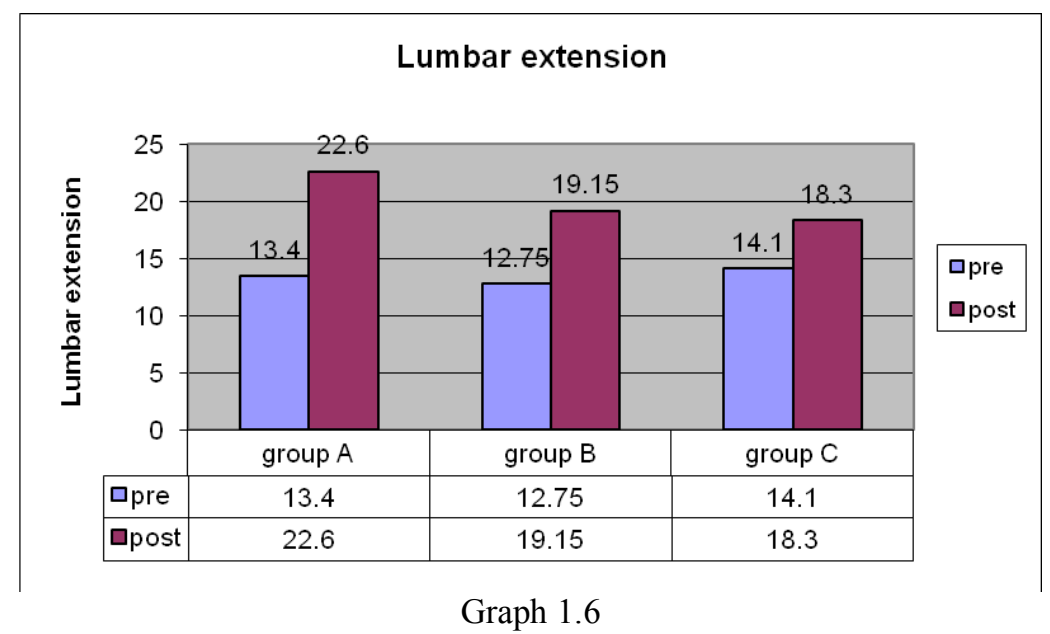

Graphical representation of Mean of lumbar flexion on left side at Pre, Post interval for the subjects of Group A, Group B and Group C

\section{Discussion}

Low back pain is an important public health, social and economic problem. It is a disorder with much possible aetiology, occurring in different groups, and also a common health condition in working population In India, occurrence of low back pain is also alarming, nearly 60 per cent of the people in India have significant back pain at some time or the other in lives. Approximately 35\% people suffer from chronic back pain, which significantly hampers their day-to-day routine (Suryapani, 1996).

There was statistically significant difference $(\mathrm{p} \leq 0.05)$ in VAS score readings and quality of life scale of the three groups when using ANOVA (table 1.1) analysed. in graph 1.1 and 1.2 group A seems to be more effective than the other two groups. The results of the study is in compliance with Taimela et al in 2000 who did a study to examine the effect of lumbar stabilization exercises on low back pain patients. They used visual analogue scale to measure pain intensity. They reported decrease in low back pain severity. In the current study the lumbar range of motion also showed different significant effect in the three groups comparing the three groups, comparing two groups one by one and comparing within the groups but in graphs 1.1, 1.2, 1.3 ,1.4, 1.5 and 1.6 it was seen that group A was more effective than the two groups. In context to the current study Hides et al in 1996 did a study that multifidus muscle recovery is not automatic after resolution of acute, first-episode low back pain. Their conclusion showed that specific, localized, holding contractions of the lumbar multifidus helped to restore symmetry of muscle size. The occurrence of a multifidus contraction was verified in the treatment session using feedback from real-time ultrasound imaging.

Another study done by Barr, Wikmar and Arvidsson (2003) to compare the stabilizing training compared with manual treatment in sub-acute and chronic low back pain further supports the findings of present study. The results showed that stabilizing training seemed to be more effective than manual treatment in terms of improvement of individuals and the reduced need for recurrent treatment periods

In the present study, there is significant difference in values of means when group A, group B and group C was compared, for VAS, QOL, and ROM. There is significant difference of paired t- test between pre and post variables of the groups for VAS and QOL, and ROM. The results show that there is significant difference of unpaired $t$ - test between group A, group B and group C for VAS, QOL and ROM. The result shows that there is significant difference in the post readings of three groups when compared together through ANOVA. Core stability exercise is based on the well-established premise that stability of the spine is dependent on the contribution of muscle. Approach aimed to train the skilled activation of the deep muscles, to train the 
integration of the deep and superficial systems, and to progress through a program of tailored functional exercises in varying environments and contexts to ensure transfer to normal activity.

\section{Conclusion}

The conclusion of the present study is that the patients of low back strain within the age group of 18-45 years are benefited more by the combination of core stabilization exercises and conventional physiotherapy rather muscle energy technique along with conventional physiotherapy.

Keywords: Low Back Pain, Core Stabilization Exercises, Muscle Energy Technique.

\section{References}

[1]. Barr, R, Wikmar, NL and Arvidsson, I. 2003. 'Stabilizing training compared with manual treatment in sub-acute and chronic lowback pain.' Man Ther, vol 8, 233-241.

[2]. Cox, JM and Trierk. 1987, 'Exercise and smoking habits in patients with and without low back pain', J Manipulative physical therapy, 224-239.

[3]. Driscoll. 2011, 'Cost associated with low back pain treatment', Asia-pac journal of public health.

[4]. Ferreira, Herbert, Maher and Refshauge. 2006,' Specific stabilisation exercise for spinal and pelvic pain', Australian journal of physiotherapy, vol 29, 79-86.

[5]. Hamilton, L. 2003, 'The effect of muscle energy technique on gross trunk range of motion', Journal osteopathic med, vol 6, 13-18

[6]. Harreby, M, Joel, A, Dehisa, Bruce, M and Nicholas, E. 1995, 'Physical med and rehabilitation', Liincott Williams and Wilkins, vol. 1, 653-679.

[7]. Hestbaek, L. 2003, 'Low back pain: A review of studies of general patient populations' European Spine Journal, Vol 12, 149165.

[8]. Hides, JA, Richardson, CA and Jull, GA, 1996, "Multifidus recovery is not automatic following resolution of active first episode of low back pain, Spine, vol. 20, .2763-2769.

[9]. Hodges, PW and Richardson, CA. 1996, 'Insufficient muscular stabilization of the lumbar spine associated with low back pain', Spine, vol 24, 2435-2448.

[10]. Huffman, LH. 2007, 'medication for acute and chronic low back pain', Ann intern med, vol 149, 505-514.

[11]. Kumar, A. 2011, 'Role of Physiotherapy in Back Pain', 23 Physio Clinic-Enhancing Physiotherapy.

[12]. Mannion, AF, Balague, F, Pellese, F and Cedrasehi, C. 2011, 'non specific low back pain', eur spine J, vol 2, 169-191.

[13]. McGill, S. M. 2001,' Low Back Stability', Performance and rehabilitation. Exercise andSportScienceReviews. Vol 17, 26-31.

[14]. Muthukrishanan, R, Shenoy, SD and Sandhu,J. 2010,' The effect of core stabilization regime and conventional physiotherapy on altered postural control parameters in patients with chronic low back pain', Sports med rehabil ther techno, 2-13.

[15]. Noelle, K, Terry, L and Hertel, G. 2003,' Muscle energy technique in lumbopelvic pain', J. Manual and manipulative therapy, vol. $17,14-18$.

[16]. Osterbauer, P and DeVita, T. 1991,' Chiropractic treatment of chronic low back', Third Annual International Conference on Spinal Manipulation. Washington. D.C, 230-1

[17]. Ruddy, T.1961, 'Osteopathic rhythmic resistive therapy', Year book of academy of alied osteopathy Indianapolis, vol 7, 56-58.

[18]. Schmitt, G. 1999,' A comparison of selected protocols during proprioceptive neuromuscular facilitation stretching', Clinical kinesiology, 16-21

[19]. Suryapani, R, 1996, "Backache, borne of modern lifestyle, The Tribune, 6 Nov, . 16.

[20]. Taimela , S, Diederich, C, Hbsch, M and Heinricy, M, 2000, "The role of physical exercise and inactivity in pain recurrence and absenteeism form work after active out patient rehabilitation for recurrent or chronic low back pain.

[21]. Tonelli, MR. 2006, 'Integrating evidence into clinical practice an alternative based aroaches', J.Eval clinical practice, vol 12, 248256.

[22]. William, C, Shiel. 2012,'Low back pain overeview',emedicine health. 